\title{
Multi-Modal Approach for Investigating Brain and Behavior Changes in an Animal Model of Traumatic Brain Injury
}

\author{
Meghan E. Heffernan, Wei Huang, Kenneth M. Sicard,2 Bernt T. Bratane, ${ }^{2}$ \\ Elif M. Sikoglu, Nanyin Zhang,, Marc Fisher, and Jean A. King ${ }^{1}$
}

\begin{abstract}
Use of novel approaches in imaging modalities is needed for enhancing diagnostic and therapeutic outcomes of persons with a traumatic brain injury (TBI). This study explored the feasibility of using functional magnetic resonance imaging (fMRI) in conjunction with behavioral measures to target dynamic changes in specific neural circuitries in an animal model of TBI. Wistar rats were randomly assigned to one of two groups (traumatic brain injury/sham operation). TBI rats were subjected to the closed head injury (CHI) model. Any observable motor deficits and cognitive deficits associated with the injury were measured using beam walk and Morris water maze tests, respectively. fMRI was performed to assess the underlying post-traumatic cerebral anatomy and function in acute ( 24 hours after the injury) and chronic ( 7 and 21 days after the injury) phases. Beam walk test results detected no significant differences in motor deficits between groups. The Morris water maze test indicated that cognitive deficits persisted for the first week after injury and, to a large extent, resolved thereafter. Resting state functional connectivity ( $\mathrm{rsFC}$ ) analysis detected initially diminished connectivity between cortical areas involved in cognition for the TBI group; however, the connectivity patterns normalized at 1 week and remained so at the 3 weeks post-injury time point. Taken together, we have demonstrated an objective in vivo marker for mapping functional brain changes correlated with injury-associated cognitive behavior deficits and offer an animal model for testing potential therapeutic interventions options.
\end{abstract}

Key words: animal model; cognition; functional connectivity; imaging; traumatic brain injury

\section{Introduction}

$\mathbf{R}$ EPORTS OF TRAUMATIC BRAIN INJURIES (TBI) are on the rise, with more than 1.5 million cases per year in the United States alone. ${ }^{1,2}$ Cognitive impairments associated with TBI affect more than 5 million persons in the United States, ${ }^{3}$ often inhibiting a person's basic ability to engage in the simplest cognitive tasks. ${ }^{4}$ Traditionally, the challenge to physicians has been to identify the effects of mild TBI in patients through a battery of neurological and imaging examinations. Unfortunately, this dual-diagnostic tool set, in its current application, is not sensitive enough to detect milder neurocognitive sequelae of TBI. ${ }^{5}$ Presenting additional hardships is the incongruent research between preclinical and clinical investigations of functional outcome. Small animal models have been developed to measure TBIassociated cognitive, motor, and somatic deficits through behavior tests, while clinical evaluation of TBI relies on structural imaging assessments to determine whether to admit or to safely discharge a patient. Neither side of the translational paradigm, however, puts a major emphasis on a multi-modal diagnostic approach. This practice can be problematic given that cognitive difficulties can persist despite lack of any observable anatomical change in brain structures related to cognitive functioning. 5,6

In this study, we have replicated an established animal model of TBI, demonstrated the feasibility of detecting cognitive deficits with behavioral tests, and cortically targeted the functional mechanisms by which the deficits occur. This approach enabled a meaningful correlation of changes in cognitive status and brain function in the acute and subacute periods after TBI. Most importantly, we developed a model with strong translational validity. Because of the frequency of closed head injury (CHI) in clinical cases, we used a CHI contusion design in an animal model of TBI, along with behavioral and imaging modalities to evaluate the extent of injury at acute and chronic time points.

Following clinical reports of commonly observed cognitive deficits and patients' desires to improve cognition, ${ }^{7}$ we have focused our analysis on disturbances within the cognitive domain.

\footnotetext{
${ }^{1}$ Department of Psychiatry, Center for Comparative NeuroImaging, University of Massachusetts Medical School, Worcester, Massachusetts.

${ }^{2}$ Department of Neurology, University of Massachusetts Memorial Medical Center, Worcester, Massachusetts.
} 
Reports of deficits in executive cognitive functioning are prevalent in brain injury cases, ${ }^{8}$ and research suggests cognitive dysfunction is most pronounced within the first weeks of injury. ${ }^{6}$ Aimed at developing a marker to map brain changes associated with behavior, this study was performed within the 3 weeks after injury. We postulated that if cognitive deficits and neuronal alterations accompany TBI, then non-invasively assessing functional connections between brain regions should be critical in evaluating the extent of injury and, possibly, recovery. In the present study, functional connectivity has been assessed by using a resting-state functional magnetic resonance imaging (rsfMRI) paradigm that has been well established through previous studies. ${ }^{9-11}$

\section{Methods}

\section{Animals}

Male Wistar rats, weighing 275-300 g, were divided into two groups (TBI, $n=7$; sham operation, $n=7$ ) and housed in pairs. The housing environment was maintained at $22-24^{\circ} \mathrm{C}$ with a $12 \mathrm{~h}$ light/ dark schedule (lights on at 06:00 and off at 18:00). Food and water were provided ad libitum. All rats were acquired and cared for in accordance with the guidelines published in the National Institutes of Health Guide for the Care and Use of Laboratory Animals.

\section{Closed head TBI model}

Closed head TBI was produced using a weight drop device, as previously described in detail. ${ }^{12}$ Briefly, the skull was exposed, and the animal's head fixed laterally between adjustable rubber-coated Plexiglas blocks to prevent head movement at the moment of impact. An incision exposing bregma was needed to maintain precision and reproducibility across animals, as well as to visually assess for skull fractures at the site of impact. A weight $(175 \mathrm{~g})$ was freely dropped $114 \mathrm{~cm}$ to strike a cylindrical polyacetal transducer rod (Delrin, tip-diameter $10 \mathrm{~mm}, 32.6 \mathrm{~g}$, e-module 29.000) placed with its tip directly on the rat's skull, posterior to bregma. The central region between bregma and lambda was aligned under the tip at an angle of 90 degrees. The transducer rod was held to prevent rebound impact immediately after the initial weight drop. Sham rats were anesthetized and surgically prepared, but were not subjected to TBI.

After the trauma or sham procedure, the incision was sutured, and animals were administered warm Ringer's lactate solution ( $5 \mathrm{~mL}$, subcutaneously) and buprenorphine $(0.05 \mathrm{mg} / \mathrm{kg}$, subcutaneously) to relieve pain. Post-procedure exclusion criteria included the presence of skull fractures and lesions seen on visual assessment or on MRI.

\section{Behavioral measurements}

Motor deficits were evaluated using the Beam walk test. Measurements of hindlimb coordination (the count of hindlimb slips off the beam surface) and navigational speed (the time lapsed from the start to end location) were recorded as the animal traversed an elevated wooden beam, $100 \mathrm{~cm}$ in length. A safe location (i.e., an enclosed box) was placed at one end of the beam as motivation for the animal to navigate across. ${ }^{13}$ Trained twice daily for a maximum of 3 min per trial, each animal was tested before the injury/sham procedure, as well as $24 \mathrm{~h}$ and 7 days post-CHI. Pre-procedure testing provided baseline performance data and habituated animals to the apparatus.

To focus on impairments in memory, cognitive abilities were measured in the Morris water maze. ${ }^{3}$ The apparatus ${ }^{14}$ and procedures ${ }^{15}$ were modified from previously established studies. To provide animals with indicators for spatial acquisition, the testing environment incorporated salient visual cues placed outside the apparatus. Animals retained information regarding the fixed location of the submerged platform with respect to the external spatial cues. Latency to the platform was recorded. Accounting for natural swimming patterns and testing bias, entry points into the pool were randomized for each trial. Four daily trials were performed for 5 consecutive days. Spatial acquisition learning was measured from day 2 to day 6 post-procedure. One week after the initial spatial acquisition, the Morris water maze was repeated in a reversal learning phase; however, the platform was relocated to the opposite quadrant of the apparatus from initial testing. ${ }^{15}$ This reversal learning phase was performed on days 15-19 post-procedure, and all methods remained consistent with the spatial acquisition phase, with the exception of the platform location.

\section{MRI}

Eleven control animals and seven TBI animals were imaged to evaluate resting-state functional connectivity (rsFC). MRI experiments were performed on a $4.7 \mathrm{~T} / 40 \mathrm{~cm}$ horizontal magnet equipped with a Biospec Bruker console (inner diameter $12 \mathrm{~cm}$ ). TBI animals were imaged at 0 (i.e., within 24 hours after the injury), 7 , and 21 days after TBI. Isoflurane was delivered at $2 \%$ with ambient air to keep animals anesthetized during imaging. T2-weighted high resolution anatomic images were acquired using Rapid Acquisition Relaxation Enhanced (RARE) sequence with relaxation time $\mathrm{TR}=2.0 \mathrm{~s}$, echo time $\mathrm{TE}=12 \mathrm{~ms}$, resolution matrix $=256 \times 256$, field of view $(F O V)=30 \mathrm{~mm} \times 30 \mathrm{~mm}$, slice number $=18$, and slice thickness $=1 \mathrm{~mm}$. Functional MRI (fMRI) incorporated multi-slice $\mathrm{T}_{2}{ }^{*}$-weighted Echo-Planar Images (EPI), acquired while at rest, to estimate rsFC. The functional imaging acquisition parameters were: $\mathrm{TR}=1000 \mathrm{~ms}, \mathrm{TE}=30 \mathrm{~ms}$, matrix size $=64 \times 64 \times 18$, and $\mathrm{FOV}=30 \mathrm{~mm} \times 30 \mathrm{~mm} \times 18 \mathrm{~mm}$. There were 200 volumes acquired each run, and six runs were repeated per imaging session.

\section{MRI data analysis}

FC (i.e., temporal coherence in spontaneous BOLD response between different brain regions) was estimated using Medical Image Visualization and Analysis (MIVA, http://ccni.wpi.edu/miva .html) and Matlab (The Mathworks Inc., Natick, MA), following a well-established computational work flow. ${ }^{9-11}$ Co-registration, based on anatomical images, was performed to a fully segmented rat brain atlas implemented in MIVA for each rat's data set.

All raw fMRI images were motion corrected using Statistical Parametric Mapping (SPM8) software (Wellcome Department of Cognitive Neurology, London, UK), and spatially filtered using a Gaussian function (FWHM $=1 \mathrm{~mm}$ ). All time courses were then 0.01-0.1 Hz band-pass filtered. The time course for each individual voxel was further corrected for head movement by regressing out the six motion parameters (translations and rotations) estimated within the motion correction step. The signal from the ventricles and white matter was estimated by averaging the time courses of all voxels within the ventricles and white matter and then was regressed out from the time courses.

The cross-correlation (CC) coefficient between regionally averaged time course of the predetermined seed region and the time course of each individual voxel was calculated. Cortical seeds were selected based on an a priori hypothesis supporting the relevance to the acquired cognitive behavioral measures. Selected anatomical seed regions included the somatosensory cortex and the infralimbic cortex. CC coefficients were transformed to $\mathrm{z}$ scores using the Fisher transformation. A connectivity map for each seed region was created for each fMRI run, and maps corresponding to the same seed across multiple runs were averaged to create the connectivity map for each animal.

\section{Statistics}

Single factor analysis of variance (ANOVA) was performed on each animal to produce comprehensive group results for all behavior measurements. Significance was determined to be $p<0.05$. 

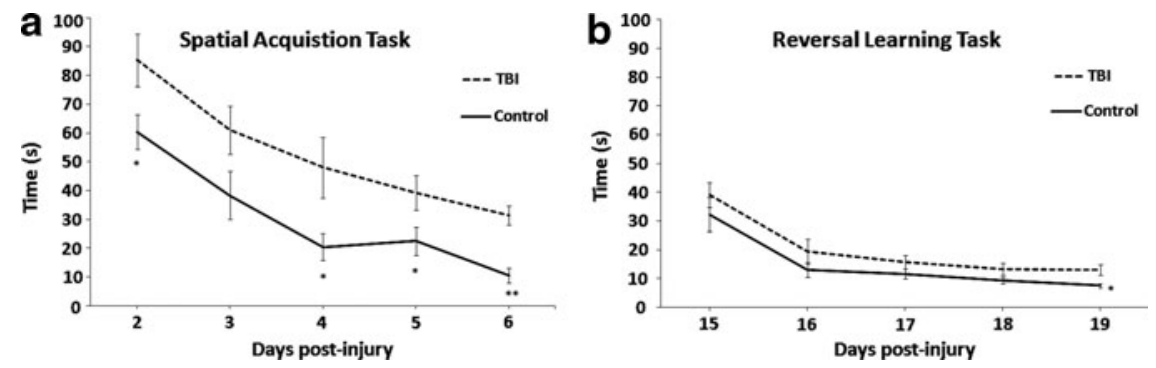

FIG. 1. Behavioral measurements-i.e., time taken to find the platform in seconds-from the Morris water maze test. (a) The spatial acquisition task was performed with the traumatic brain injury (TBI) and control groups for 5 days, starting post-surgery day 2. Significant intergroup differences were observed on all days, with the exception of the second day of testing. (b) Two weeks postinjury, a reversal learning task was performed with the same rats. Significant intergroup difference was observed on the last day of testing.

For each seed region, a composite connectivity map was generated using a one sample $t$ test across subjects, to create group result maps of $\mathrm{FC}$, thresholded at $p<0.05$. False positives were corrected using FDR (false discovery rate) in SPM8 at $p<0.05$.

In addition, average $\mathrm{CC}$ coefficients in different regions of interest (ROIs) were calculated to quantify regional connectivity change. Group difference of regional averaged CC coefficients was evaluated using two-sample $t$ tests.

\section{Results}

\section{Beam walk test}

Average time to traverse the beam did not reveal significant intergroup differences at any of the three time points (baseline, $[\mathrm{F}(1,12)=0.04, p>0.5] ; 24 \mathrm{~h}$ post-injury, $[\mathrm{F}(1,12)=2.99, p>0.1]$; 7 days post-injury, $[\mathrm{F}(1,12)=0.02, p>0.5])$. Likewise, hindlimb slips were statistically similar between groups when measured at either post-injury time point (baseline, $[\mathrm{F}(1,12)=2.4, p>0.1] ; 24 \mathrm{~h}$ post-injury, $[\mathrm{F}(1,12)=4.29, \quad p>0.05] ; 7$ days post-injury, $[\mathrm{F}(1,12)=0.37, p>0.5])$. The results detected no significant motor deficits associated with the brain injury.

\section{Morris water maze test}

Single factor ANOVA revealed significant intergroup differences as demonstrated in Figure 1a. Shorter latency to the platform was observed in control animals, relative to TBI counterparts, during spatial acquisition testing on day $2[\mathrm{~F}(1,12)=5.17, p=0.04]$, day $4[\mathrm{~F}(1,12)=5.72, p=0.03]$, day $5[\mathrm{~F}(1,12)=4.84, p=0.04]$, and day $6[\mathrm{~F}(1,12)=32.06, p=0.0001]$ post-surgery. Results of day 3 testing yielded a trend toward significance in behavioral performance $[\mathrm{F}(1,12)=3.69, p=0.07]$. Initial differences seen between groups on post-surgery day 2 are representative of cognitive deficits $48 \mathrm{~h}$ post-injury and cannot be explained as differences in motor abilities given results of the beam walk test. Analysis of reversal learning (Fig. 1b) revealed significant intergroup difference on the last day of testing $[\mathrm{F}(1,12)=7.36, p=0.01]$, with slower performance by TBI animals, but did not reach statistical significance on the first 4 days.

\section{FC}

FC patterns did not demonstrate significant differences between the TBI and the control groups, $24 \mathrm{~h}, 7$ days, and 21 days postsurgery, when the seed was placed at the somatosensory cortex. This result was consistent with the beam walk test, suggesting there was no motor deficit in TBI animals.
Figure 2 shows the composite FC maps using infralimbic cortex as the seed, for the control group (Fig. 2a) as well as the TBI group $24 \mathrm{~h}$ (Fig. 2b), 7 days (Fig. 2c), and 21 days (Fig. 2d) post-surgery. Compared with controls, diminished rsFC in TBI animals, particularly between infralimbic and the anterior cingulate cortex (ACC), was observed at the initial imaging time point ( $24 \mathrm{~h}$ post-surgery) and normalized thereafter to a similar strength as controls by day 7 post-surgery. This normalization in connectivity remained until day 21.

Figure 3 illustrates the average $\mathrm{CC}$ coefficient between the infralimbic cortex and the ACC, characterizing the strength of the connectivity, thresholded with CC of 0.2. Compared with the control group, connectivity between the infralimbic cortex and ACC was impaired in the TBI group only for the first $24 \mathrm{~h}$ after injury $(\mathrm{t}(16)=4.18, p=0.001)$. TBI intragroup comparison showed significant alterations in connectivity on day $7(\mathrm{t}(12)=-4.1$, $p=0.006$ ) compared with day 1 post-injury, and remained similar to control level on day 21 .

\section{Discussion}

Standard neuroradiological techniques have the potential to detect anatomical injuries associated with TBI; however, many patients with mild or moderate TBI experience functional deficits in cognition, regardless of the existence or absence of structural lesions. For this reason, we chose an animal model of mild TBI without any observable abnormalities in serial MRI, but displayed behavioral, specifically cognitive, challenges. ${ }^{12}$ In the present study, our TBI animal model demonstrated cognitive deficits that persisted up to 1 week post-injury, with a relative disappearance of intergroup difference over the subsequent 2 weeks. In addition, functional connectivity analysis detected diminished correlation between time courses from different cortical areas $24 \mathrm{~h}$ postsurgery. Significant improvement in connectivity, however, returned these measures to the control level at day 7 and day 21 post-surgery. This multi-modal approach enabled us to identify functional brain changes associated with quantified behavioral deficits in an injury model absent of any anatomical abnormalities.

Despite the absence of deficits in behavior performance of animals with TBI from 1 week to 3 weeks post-injury, modest differences in the reversal learning task were exhibited at the end of testing. In clinical cases, regardless of the existence or absence of structural lesions, patients with TBI often report cognitive difficulties, especially in circumstances requiring them to remain ontask for an extended period. Referred to as "cognitive fatigue" or deficits in cognitive endurance, one hypothesis for this 

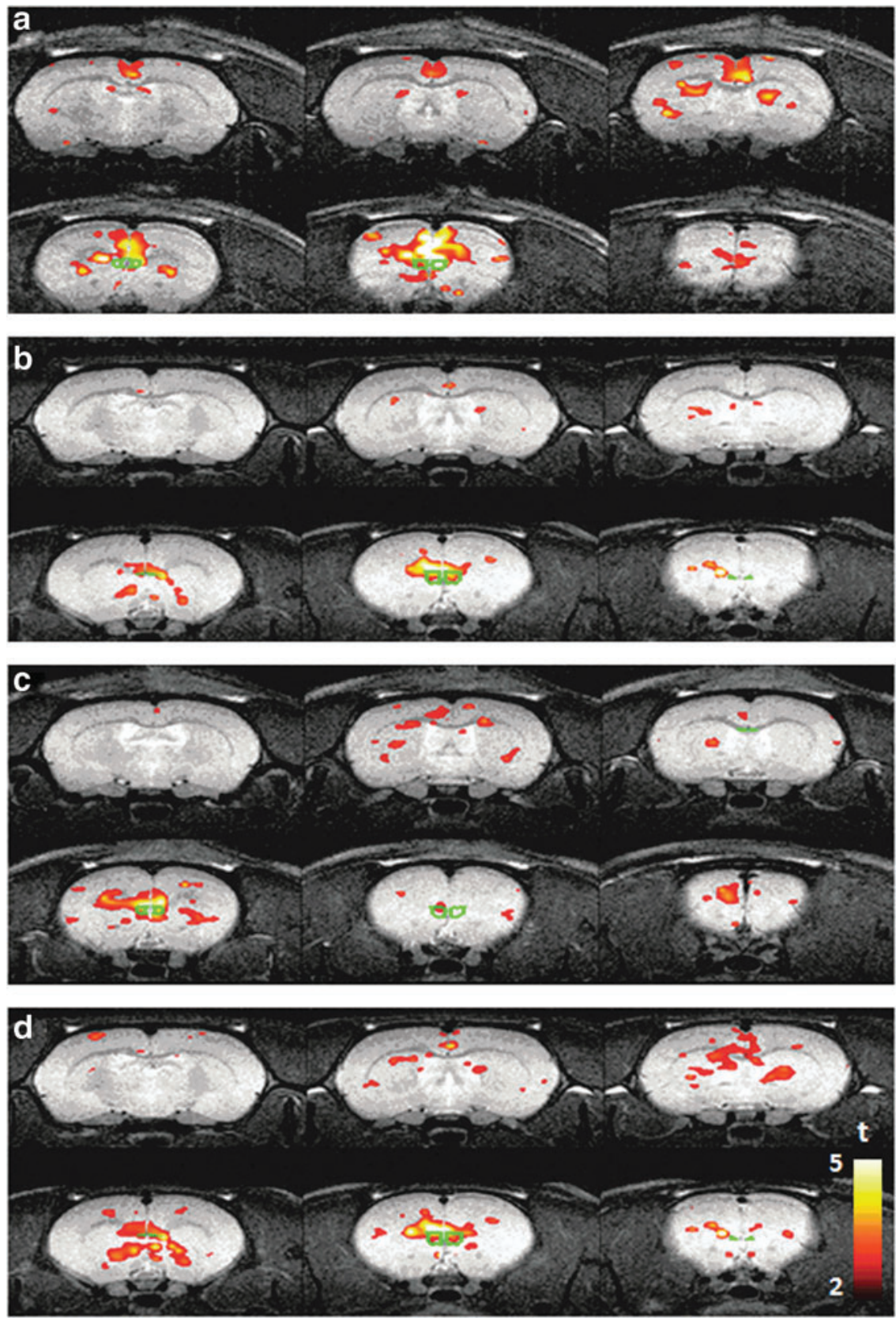

FIG. 2. The functional connectivity map from the seed of infralimbic cortex. (a) Control group within 24h post-surgery; (b) TBI group within $24 \mathrm{~h}$ post-injury; (c) TBI group 7 days post-injury; (d) TBI group 21 days post-injury. The green outline denotes the location of the seed region. The connectivity maps were normalized 7 days post-injury to the level observed in the control group. Color image is available online at www.liebertpub.com/neu

phenomenon is that patients do not experience significant decreased cognitive performance over time, but instead experience persistent fatigue during a task necessitating sustained cognitive effort for a prolonged period. ${ }^{16,17}$ When an extended task coincides with a distracting environment, fatigue is thought to be intensified. ${ }^{16}$

The f5-day reversal learning phase in rats may serve as a proxy for a prolonged cognitive task in humans. Therefore, the theory of cognitive fatigue may provide a mechanistic basis for the significant intergroup difference observed on only the last day of testing. Given our data, we speculate that rats with TBI potentially began cognitive recovery in the week between spatial acquisition and reversal learning, as explained by the lack of significance in behavioral measures on days 15-18, but showed a deficit in cognitive endurance by the fifth day of consecutive testing. The requirement to consistently recall information of the platform location based on numerous salient visual cues may have impacted the cognitive endurance of the recovering animals with TBI.

As an addendum to the traditional behavior measurements, we used rsFC to assess deficits in intrinsic brain connectivity, a technique that is novel to animal model investigations of TBI. Typically, functional imaging investigations of TBIs study widespread deficits in evoked neuronal activity. ${ }^{18,19}$ Notwithstanding the importance of targeting these general task-related activations, 


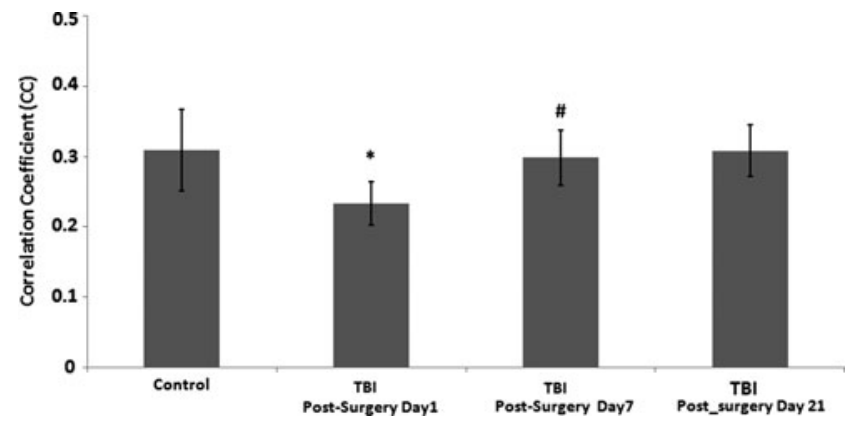

FIG. 3. Average correlation coefficients (CCs) across 11 control rats and 7 rats with traumatic brain injury (TBI), representing the strength of functional connections between the seed region, i.e., infralimbic cortex, and the anterior cingulate cortex (ACC). Threshold CC was set to 0.2. Compared with the control group, connectivity between the infralimbic cortex and ACC significantly weakened in the TBI group within 24 hours after injury $(* \mathrm{t}(16)=4.18, p=0.001)$. TBI intragroup comparison between days 1 and 7 showed statistically different connectivity $\left({ }^{\#} \mathrm{t}(12)=\right.$ $-4.1, p=0.006$ ) and on day 21 post-surgery remained normalized to the level of the control group.

previous techniques were restricted to providing information on cortical activations within, rather than between, particular brain regions. A series of FC studies have demonstrated that patterned communications exist within various brain networks during resting and passive task states. ${ }^{20}$ During these states, distributed brain regions within functional-anatomic networks spontaneously increase and decrease intrinsic activity together. ${ }^{21}$ Temporally correlated, these spontaneous fluctuations map intrinsic brain activity within and between different brain networks. Recently added ${ }^{6,22,23}$ to the conventional MRI techniques used in clinical TBI studies, ${ }^{24-27}$ FC has just begun to investigate potential damage to specific interregional neural connections generated by mild to moderate TBI. ${ }^{6,28,29}$

rsFC imaging, in the present study, detected discrete differences in connectivity strength between selective brain areas of the rats with TBI from the time of initial injury to the study end-point. Consistent with our cognitive behavioral measurement, disrupted connectivity was observed immediately after injury. Interestingly, results at the 3-week time point showed normalized cortical connectivity in the rats with TBI to that of the control animals, whereas behavior results still showed slowed escape latency. Similarities in post-surgery days 7 and 21 imaging results suggest that neuroadaptation in rats with TBI can restore similar connection strength in brain regions involved in cognitive tasks, ${ }^{4}$ despite performance differences, relative to controls, on the first 4 days of each testing phase.

Taken together, we speculate that the return to baseline (control) connectivity between cortical regions at later imaging time points, sans any external therapeutic intervention, may be indicative of neuroplasticity changes post-injury. That is, the observed dynamic changes, from weakened to normalized connectivity in comparison with control animals and slowed to almost normalized behavior performance, may be the result of neuroplastic processes responsible for restoring disrupted patterns of communication between neural circuits, as early as 1 week post-injury. Indeed, rsFC may serve as a valuable biomarker of injury and restoration of function in a manner similar to that noted in the reorganization of certain connections after peripheral nerve injury. ${ }^{30}$

There are several limitations to this study. While our concussion device was guided but not mechanistically controlled, thus pre- senting a potential constraint to the intragroup reproducibility, we did not observe significant intragroup differences in any of the acquired measures. With regard to intergroup comparison, control animals were imaged once based on the assumption that consistent results will be obtained by repeated imaging, because the reliability of rsFC measurement over time has been widely confirmed with test-retest designs. Further, the reliability of rsFC in anesthetized animals is confirmed by Liu and colleagues ${ }^{31}$ who reported a strong neurovascular coupling in isoflurane-anesthetized rats, suggesting rsFC measured by fMRI in isoflurane anesthetized rats was of neural origin. Isoflurane is a vasodilator that may change the baseline blood flow level in the brain; however, rsfMRI measurement is not sensitive to baseline blood flow level, because it assesses the temporal coherence of spontaneous fluctuations of the fMRI signal, rather than its absolute amplitude. Therefore, the use of isoflurane should not have significant impact on rsfMRI measurement and the imaging of control animals only once should not change rsFC measurements. Finally, because we do not have the statistical power to perform statistical comparison for individual voxels and the main objective of this study was to examine changes of rsFC between brain regions (i.e., ROIs), data were statistically compared on the ROI level and not voxel-by-voxel basis.

\section{Conclusion}

We examined a $\mathrm{CHI}$ animal model to establish the feasibility of using behavior and FC assessments as a biomarker for dynamic changes associated with cognitive progression in TBI. Twofold in relevance, our findings present an objective in vivo marker for mapping functional brain changes correlated with injury-associated cognitive behavior deficits and offer an animal model, strong in translational validity, for testing potential therapeutic interventions options targeted at preclinical TBI.

\section{Acknowledgments}

We would like to thank Dr. Michael Whalen from Massachusetts General Hospital for his support and collaboration during this study.

\section{Author Disclosure Statement}

No competing financial interests exist.

\section{References}

1. Gao, X., and Chen, J. (2011). Mild traumatic brain injury results in extensive neuronal degeneration in the cerebral cortex. J. Neuropathol. Exp. Neurol. 70, 183-191.

2. Pleasant, J.M., Carlson, S.W., Mao, H., Scheff, S.W., Yang, K.H., and Saatman, K.E. (2011). Rate of neurodegeneration in the mouse controlled cortical impact model is influenced by impactor tip shape: implications for mechanistic and therapeutic studies. J. Neurotrauma 28, 2245-2262.

3. Davis, A.R., Shear, D.A., Chen, Z., Lu, X.C., and Tortella, F.C. (2010). A comparison of two cognitive test paradigms in a penetrating brain injury model. J. Neurosci. Methods 189, 84-87.

4. Van Boven, R.W., Harrington, G.S., Hackney, D.B., Ebel, A., Gauger, G., Bremner, J.D., D’Esposito, M., Detre, J.A., Haacke, E.M., Jack, C.R., Jr., Jagust, W.J., Le Bihan, D., Mathis, C.A., Mueller, S., Mukherjee, P., Schuff, N., Chen, A., and Weiner, M.W. (2009). Advances in neuroimaging of traumatic brain injury and posttraumatic stress disorder. J. Rehabil. Res. Dev. 46, 717-757.

5. Newcombe, V., Chatfield, D., Outtrim, J., Vowler, S., Manktelow, A., Cross, J., Scoffings, D., Coleman, M., Hutchinson, P., Coles, J., Carpenter, T.A., Pickard, J., Williams, G., and Menon, D. (2011). Mapping traumatic axonal injury using diffusion tensor imaging: correlations with functional outcome. PLoS One 6, e19214. 
6. Mayer, A.R., Mannell, M.V., Ling, J., Gasparovic, C., and Yeo, R.A (2011). Functional connectivity in mild traumatic brain injury. Hum. Brain Mapp. 32, 1825-1835.

7. Corrigan, J.D., Whiteneck, G., and Mellick, D. (2004). Perceived needs following traumatic brain injury. J. Head Trauma Rehabil. 19, 205-216.

8. Riese H, Hoedemaeker, M., Brouwer, W.H., Mulder, L.J.M., Cremer, R., and Veldman, J.B.P. (1999). Mental fatigue after very severe closed head injury: Sustained performance, mental effort and distress at two levels of workload in a driving simulator. Neuropsychol. Rehabil. 9, 189-205.

9. Zhang, N., Rane, P., Huang, W., Liang, Z., Kennedy, D., Frazier, J.A., and King, J. (2010). Mapping resting-state brain networks in conscious animals. J. Neurosci. Methods 189, 186-196.

10. Liang, Z., King, J., and Zhang, N. (2012). Anticorrelated resting-state functional connectivity in awake rat brain. Neuroimage 59, 11901199.

11. Liang, Z., King, J., and Zhang, N. (2011). Uncovering intrinsic connectional architecture of functional networks in awake rat brain. J. Neurosci. 31, 3776-3783.

12. Henninger, N., Dutzmann, S., Sicard, K.M., Kollmar, R., Bardutzky, J., and Schwab, S. (2005). Impaired spatial learning in a novel rat model of mild cerebral concussion injury. Exp. Neurol. 195, 447-457.

13. Virley, D., Beech, J.S., Smart, S.C., Williams, S.C., Hodges, H., and Hunter, A.J. (2000). A temporal MRI assessment of neuropathology after transient middle cerebral artery occlusion in the rat: correlations with behavior. J. Cereb. Blood Flow Metab. 20, 563-582.

14. Morris, R. (1984). Developments of a water-maze procedure for studying spatial learning in the rat. J. Neurosci. Methods 11, 47-60.

15. Vorhees, C.V., and Williams, M.T. (2006). Morris water maze: procedures for assessing spatial and related forms of learning and memory. Nat. Protoc. 1, 848-858.

16. Lux, W.E. (2007). A neuropsychiatric perspective on traumatic brain injury. J. Rehabil. Res. Dev. 44, 951-962.

17. Kohl, A.D., Wylie, G.R., Genova, H.M., Hillary, F.G., and Deluca, J. (2009). The neural correlates of cognitive fatigue in traumatic brain injury using functional MRI. Brain Inj. 23, 420-432.

18. Perlstein, W.M., Cole, M.A., Demery, J.A., Seignourel, P.J., Dixit, N.K., Larson, M.J., and Briggs, R.W. (2004). Parametric manipulation of working memory load in traumatic brain injury: behavioral and neural correlates. J. Int. Neuropsychol. Soc. 10, 724-741.

19. McAllister, T.W., Saykin, A.J., Flashman, L.A., Sparling, M.B., Johnson, S.C., Guerin, S.J., Mamourian, A.C., Weaver, J.B., and Yanofsky, N. (1999). Brain activation during working memory 1 month after mild traumatic brain injury: a functional MRI study. Neurology 53, 1300-1308.

20. Gusnard, D.A., Akbudak, E., Shulman, G.L., and Raichle, M.E. (2001). Medial prefrontal cortex and self-referential mental activity: relation to a default mode of brain function. Proc. Natl. Acad. Sci. U. S. A. 98, 4259-4264.

21. Fox, M.D., Snyder, A.Z., Vincent, J.L., Corbetta, M., Van Essen, D.C., and Raichle, M.E. (2005). The human brain is intrinsically organized into dynamic, anticorrelated functional networks. Proc. Natl. Acad. Sci. U. S. A. 102, 9673-9678.

22. Hillary, F.G., Slocomb, J., Hills, E.C., Fitzpatrick, N.M., Medaglia, J.D., Wang, J., Good, D.C., and Wylie, G.R. (2011). Changes in resting connectivity during recovery from severe traumatic brain injury. Int. J. Psychophysiol. 82, 115-123.

23. Bonnelle, V., Leech, R., Kinnunen, K.M., Ham, T.E., Beckmann, C.F., De Boissezon, X., Greenwood, R.J., and Sharp, D.J. (2011). Default mode network connectivity predicts sustained attention deficits after traumatic brain injury. J. Neurosci. 31, 13442-13451.

24. Scheid, R., Preul, C., Gruber, O., Wiggins, C., and von Cramon, D.Y. (2003). Diffuse axonal injury associated with chronic traumatic brain injury: evidence from $\mathrm{T} 2 *$-weighted gradient-echo imaging at $3 \mathrm{~T}$. AJNR Am. J. Neuroradiol. 24, 1049-1056.

25. Johnston, K.M., Ptito, A., Chankowsky, J., and Chen, J.K. (2001) New frontiers in diagnostic imaging in concussive head injury. Clin. J. Sport Med. 11, 166-175.

26. Belanger, H.G., Vanderploeg, R.D., Curtiss, G., and Warden, D.L. (2007). Recent neuroimaging techniques in mild traumatic brain in jury. J. Neuropsychiatry Clin. Neurosci. 19, 5-20.

27. Beauchamp, M.H., Ditchfield, M., Babl, F.E., Kean, M., Catroppa, C., Yeates, K.O., and Anderson, V. (2011). Detecting traumatic brain lesions in children: CT versus MRI versus susceptibility weighted imaging (SWI). J. Neurotrauma 28, 915-927.

28. Stevens, M.C., Lovejoy, D., Kim, J., Oakes, H., Kureshi, I., and Witt S.T. (2012). Multiple resting state network functional connectivity abnormalities in mild traumatic brain injury. Brain Imaging Behav. 8, 293-318.

29. Slobounov, S.M., Gay, M., Zhang, K., Johnson, B., Pennell, D., Sebastianelli, W., Horovitz, S., and Hallett, M. (2011). Alteration of brain functional network at rest and in response to YMCA physical stress test in concussed athletes: RsFMRI study. Neuroimage 55, 1716-1727.

30. Pawela, C.P., Biswal, B.B., Hudetz, A.G., Li, R., Jones, S.R., Cho, Y.R., Matloub, H.S., and Hyde, J.S. (2010). Interhemispheric neuroplasticity following limb deafferentation detected by resting-state functional connectivity magnetic resonance imaging (fcMRI) and functional magnetic resonance imaging (fMRI). Neuroimage 49, 2467-2478.

31. Liu, X., Zhu, X.H., Zhang, Y., and Chen, W. (2011). Neural origin of spontaneous hemodynamic fluctuations in rats under burst-suppression anesthesia condition. Cereb. Cortex 21, 374-384.

Address correspondence to:

Jean A. King, PhD

University of Massachusetts Medical School 55 Lake Avenue North Worcester, MA 01655

E-mail: Jean.King@umassmed.edu 